\title{
Strength training and protein supplementation improve muscle mass, strength, and function in mobility-limited older adults: a randomized controlled trial
}

\author{
Sigve Nyvik Aas ${ }^{1}$ (D) . Olivier Seynnes ${ }^{1} \cdot$ Haakon B. Benestad ${ }^{2} \cdot$ Truls Raastad $^{1}$
}

Received: 26 March 2019 / Accepted: 28 May 2019

(c) Springer Nature Switzerland AG 2019

\begin{abstract}
Background Adaptation to strength training in very old mobility-limited individuals is not fully characterized. Therefore, the aim of this study was to perform a thorough investigation of the adaptation to a lower body strength training regime in this population, with particular emphasis on the relationship between changes in selected variables.

Methods Twenty-two mobility-limited older men and women ( $85 \pm 6$ years) were randomized to either a group performing $30 \mathrm{~min}$ of heavy-load strength training three times a week, with daily protein supplementation, for 10 weeks (ST), or a control group. End points were leg lean mass assessed by DXA, muscle thickness assessed by ultrasound, isometric and dynamic strength, rate of torque development, and functional capacity.

Results Leg lean mass increased from baseline in ST $(0.7 \pm 0.3 \mathrm{~kg})$, along with increased thickness of vastus lateralis $(4.4 \pm 3.2 \%)$, rectus femoris $(6.7 \pm 5.1 \%)$, and vastus intermedius $(5.8 \pm 5.9 \%)$. The hypertrophy was accompanied by improved knee extensor strength (20-23\%) and functional performance (7-11\%). In ST, neither the change in leg lean mass nor muscle thickness correlated with changes in muscle strength. However, a strong correlation was observed between the change in isometric strength and gait velocity $(r=0.70)$.

Conclusions The mismatch between gains in muscle size and strength suggests that muscle quality-related adaptations contributed to the increases in strength. The correlations observed between improvements in strength and function suggests that interventions eliciting large improvements in strength may also be superior in terms of functional gains in this population.
\end{abstract}

Keywords Resistance exercise $\cdot$ Frailty $\cdot$ Sarcopenia $\cdot$ Hypertrophy $\cdot$ Mobility limited $\cdot$ Skeletal muscle

\section{Introduction}

Muscle strength is associated with functional performance in older adults [1] and various tasks of daily living are therefore hampered by the rapid decline in strength at very high age. The Short Physical Performance Battery (SPPB) is commonly used to assess functional capacity, and individuals

Electronic supplementary material The online version of this article (https://doi.org/10.1007/s40520-019-01234-2) contains supplementary material, which is available to authorized users.

Sigve Nyvik Aas

s.n.aas@nih.no

1 Department of Physical Performance, Norwegian School of Sport Sciences, Oslo, Norway

2 Section of Anatomy, Institute of Basic Medical Sciences, University of Oslo, Oslo, Norway with a score of 10 or less out of maximum 12 may be categorized as mobility limited. These individuals represent a group of particular interest, because small-to-moderate limitations in functional status assessed by SPPB is associated with higher odds of losing the ability to walk $400 \mathrm{~m}$ 3 years later [2].

It is established that heavy-load strength training, alone or in combination with protein supplementation, can improve muscle mass, strength, and function in healthy older adults [3-5]. In contrast, studies in very old individuals with certain mobility limitations are scarce and report discrepant findings. Gains in lean mass are observed by some [6], but not all [7], and information about the hypertrophic adaptation within specific muscles is lacking. Training effects on knee extension strength measured as one-repetition maximum (1RM) vary greatly, from 25 to 200\% [6-9]. Isometric strength gains are substantially lower, ranging from $\sim 5$ to $37 \%[7,8]$. Findings are also inconsistent with respect 
to functional improvements [7, 9]. Therefore, the extent to which training-induced gains in muscle mass and size are related to improvements in strength and function is still poorly understood in this population, and studies combining different methods to assess changes in muscle mass, strength and function are therefore needed. Moreover, to improve the basis for investigating such relationships, it might be beneficial to include a protein supplement to optimize the gains in muscle mass [10].

Hence, the aim of the present investigation was to conduct a thorough examination of the adaptation to strength training and protein supplementation in very old mobility-limited individuals. By complementing leg lean mass changes with ultrasound measurements of quadriceps femoris, we aimed to precisely characterize changes in muscle mass. Moreover, both dynamic and isometric strength testing, as well as several functional tests were performed, to investigate the relationships between improvements in muscle function and functional performance. We hypothesized that strength training and protein supplementation would elicit increases in both leg lean mass and muscle thickness, but that these increases would be exceeded by greater improvements in muscle strength. Besides, we hypothesized that muscle hypertrophy would show stronger correlations with changes in isometric than dynamic strength. Lastly, we hypothesized that improvements in both dynamic and isometric strength would correlate with improvements in functional performance.

\section{Methods and design}

The study was conducted from August to December 2015 in Oslo, Norway. Twenty-two older men and women aged between 73 and 92 years [ $85 \pm 6$ years; mean \pm standard deviation (SD)] were recruited through advertisement in the local community and visits at a local senior activity center. Upon inclusion, subjects were screened using the Short Physical Performance Battery (SPPB). The SPPB consists of timed standing balance, gait speed, and timed chair-rise assessments [11]. Performance for each of these tasks is scored between 0 and 4 , with a summary score of 0-12. Participants with an SPPB score $\leq 10$ were included. In addition, individuals using aids such as walkers, canes or Nordic walking sticks in their daily life were included, regardless of SPPB score. Participants were excluded if they had significant cognitive impairment, unstable chronic illness, cardiac arrhythmia, had experienced a fracture of a lower extremity during the last year, were smokers, or had other musculoskeletal problems making testing and training impossible.

The study complied with the standards set by the Declaration of Helsinki. The nature and goals of the study were thoroughly explained, and all participants provided a written informed consent. The study was evaluated by the Regional Committee for Medical and Health Research Ethics, Section C, South East Norway. The study data storage methods were in accordance with The Norwegian Centre for Research Data. The study was retrospectively registered at clinicaltrials.gov as NCT03723902 (URL: https://clinicaltrials.gov/ show/NCT03723902). After baseline assessment, stratified randomization was performed on ranked baseline knee extensor strength (normalized to body weight), five times chair-rise and stair-climbing performance. Randomization was performed by a researcher not involved in the study, using an online random allocation software. Participants were assigned in a 1:1 ratio to a group performing heavyload strength training three times per week and receiving daily protein supplementation, both for 10 weeks (ST), or a control group continuing their normal daily activities and dietary habits (CON) (Fig. 1).

\section{Training}

Training of participants in ST was carried out at a local senior activity center. The strength training protocol was a supervised progressive program, performed three times per week for 10 weeks. The training was performed in groups of two to three participants, and each session took approximately $30 \mathrm{~min}$ to complete, including warm-up. Sessions were usually performed Mondays, Wednesdays and Fridays, where Mondays and Fridays were repetition maximum (RM) sessions. On Wednesdays, the load was adjusted to approximately 80-90\% of RM loads. Each session included leg press, knee extension, and adapted one-leg squat. In the latter exercise, subjects stood sideways toward a wall bar for support (one hand), with the opposite leg on a step platform (initial height of $20 \mathrm{~cm}$ ). From this position, subjects extended their knee and hip, before returning to the starting position. After a given number of repetitions, the participant turned around $180^{\circ}$, to perform the exercise for the other leg. Progression was achieved by gradually increasing the height of the step platform and by adding weight (dumbbell in the arm not used for support). For all three exercises, subjects were instructed to use 1-2 s both on the concentric and eccentric phase. The participants performed one to three sets of 12 repetitions at submaximal loads the first week, and from week 2-10 subjects gradually progressed from three sets of 12RM to three to four sets of 6RM (Table 1). Each set was followed by approximately 2 -min rest periods. Strength training sessions were preceded by a lower extremity 3-min warm-up exercise, using a step platform. 


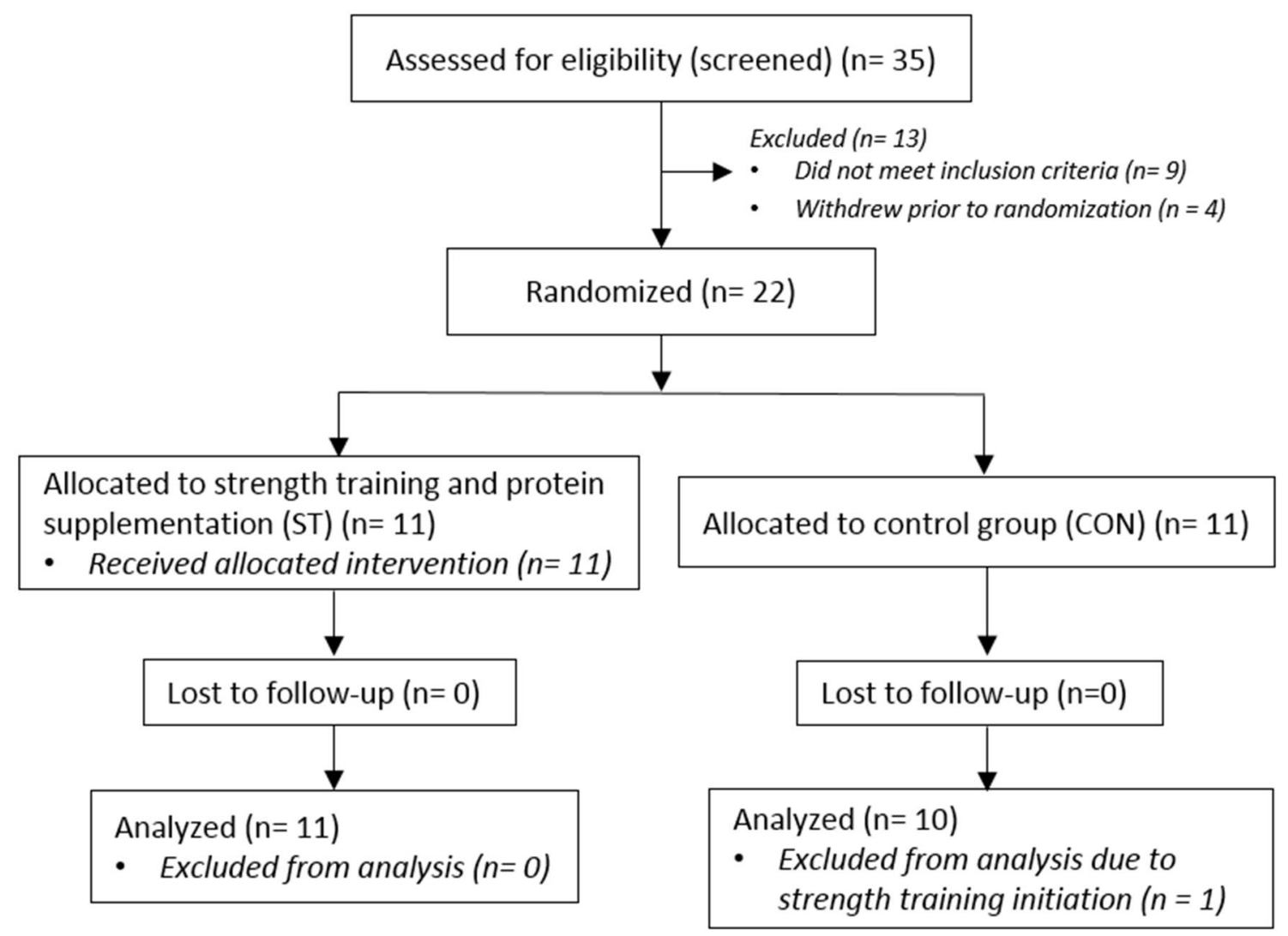

Fig. 1 Participants' flowchart

\section{Supplementation}

Participants in ST received $34 \mathrm{~g}$ of milk protein each day, divided in two equal servings [each contained $17 \mathrm{~g}$ of protein, $18 \mathrm{~g}$ of carbohydrate, $1 \mathrm{~g}$ fat, giving $149 \mathrm{kcal}$ $(627 \mathrm{~kJ})]$. Participants consumed one supplement in the morning and one in the evening. On training days, subjects were instructed to consume one of the servings within $2 \mathrm{~h}$ after training. Participants were encouraged to continue their regular food routines in addition to the supplements.

\section{Tests and measurements}

All tests were monitored by the same investigator before and after the intervention period. Neither participants nor test personnel were blinded during testing. Analyses of ultrasound images were performed in a blinded manner. All testing took place at the Norwegian School of Sport Sciences.

\section{Diet assessment}

24-h diet recall interviews were performed to evaluate whether the supplement had changed the participants' diet. Two interviews were conducted before, and two toward the end of the intervention period.

\section{Body composition}

\section{Dual energy X-ray absorptiometry}

Fat and lean mass was assessed with a DXA scan after an overnight fast. Regional analyses were performed to investigate leg lean mass (primary outcome). The test-retest reliability for measuring leg lean mass is high in our laboratory $(\mathrm{CV}<1.7 \%)$. 


\section{Quadriceps thickness}

Muscle thickness was measured in the vastus lateralis, rectus femoris, and vastus intermedius muscles of the dominant leg, with a 50-mm linear probe $(5-12 \mathrm{MHz})$ connected to a Philips HD11 XE ultrasound apparatus (Royal Philips Electronics, Amsterdam, the Netherlands). Scans of vastus lateralis were obtained at $40 \%$ of the distance between the knee joint and the greater trochanter, and halfway between the iliac crest and the myotendinous junction for rectus femoris. Vastus intermedius was measured on the same image as vastus lateralis, where the latter was thickest in the medio-lateral direction. Probe position was recorded for each measurement on transparent, acetate paper positioned over the thigh.

Several images were obtained for each muscle at each registration time point, and the three best images were chosen for analysis, which was performed using OsiriX v5.5.1 (Bernex, Switzerland). The shortest distance between the upper and lower aponeurosis was measured at 25, 50, and $75 \%$ of the width of the field of view. Muscle thickness was calculated as the average of these measurements across images.

\section{Muscle strength}

Maximal strength was assessed with a one-repetition maximum (1RM) in knee extension. After a general warm-up (5 min bicycling), a specific warm-up/preparation procedure was performed using loads corresponding to 50, 70, 80 , and $90 \%$ of the expected $1 \mathrm{RM}$ - conducting $10,6,3$, and 1 repetitions, respectively. Optimally, two to five attempts were used to establish 1RM. The loads could be adjusted in steps as low as $0.5 \mathrm{~kg}$. The left and right legs were tested interchangeably (unilateral), and each leg rested for approximately 3 min between attempts. The range of motion was strictly controlled.

Isometric maximal voluntary contraction (MVC) was also tested for the knee extensors. The participants were securely strapped to the testing chair, with an angle of $90^{\circ}$ in the hip and knee joints ( $0^{\circ}$ corresponding to full extension). The lever arm of the knee extension device with an attached strength gauge (HBM U2AC2, Darmstadt, Germany) was adjusted so that the contact point with the participant's calf was just proximal to the line of the medial malleolus. The participants performed three 5-s attempts and were instructed to reach maximum torque (Newton meter- $-\mathrm{Nm}$ ) as rapidly as possible. This was done to obtain a measure of the rate of torque development (RTD), both in relative terms $\left(\mathrm{RTD}_{\max }\right.$ /maximal torque) and absolute terms (torque at $100 \mathrm{~ms}$ ). The left and right legs were tested interchangeably, with $60 \mathrm{~s}$ of rest between each attempt. The best result of three trials per leg was used in the analyses. 


\section{Functional performance}

\section{Five times chair rise}

Participants were asked to rise from a chair to a full stand five times as fast as possible. Subjects were instructed to keep their hands crossed on their chest. If they were not able to stand up without using their hands, the use of hands was allowed, and performed the same way at both registration time points. Time was measured with a pressure sensor (TC-Start Pod and Touch Pad, Brower Timing Systems, Draper, USA). The best time of the two trials was used in the analyses.

\section{Stair-climbing test}

Participants were asked to climb a stair with 20 steps as fast as possible, without running. Time was assessed by photocells (Brower Timing Systems, Draper, USA). Steps were $18 \mathrm{~cm}$ high and 30 long (horizontal length). The total height was $3.6 \mathrm{~m}$ and the total length was $6 \mathrm{~m}$. The participants were encouraged to climb the stairs without using the handrail, but were allowed to do so if they felt it was necessary. If so, hands were used at both registration time points. The best time of the two trials was used in the analyses.

\section{Habitual gait velocity}

Participants were asked to walk $10 \mathrm{~m}$ at their habitual gait velocity. Photocells (Brower Timing Systems, Draper, USA) were placed to measure the time between 2 and $8 \mathrm{~m}$. The mean time of three trials was used in the analyses.

\section{Statistical analyses}

Leg lean mass was the primary outcome. The sample size calculation was based on values obtained from a previous study [10] and on unpublished results from our own laboratory. Using an online calculator (clincalc.com), power calculations indicated that 22 participants would be needed to identify a difference of $0.5 \mathrm{~kg}$ between the ST and CON for leg lean mass (alpha set at 0.05, beta at 0.20), with an expected standard deviation (SD) of $0.4 \mathrm{~kg}$. Results for the functional tests (five times chair rise, stair climbing, and habitual gait velocity) were not normally distributed (D'Agostino and Pearson omnibus normality test) and were therefore log transformed prior to statistical analysis. Between-group differences at baseline were assessed with an unpaired Student's $t$ test. A two-way ANOVA and Sidak's multiple comparison test were used to evaluate the effect of time and group for variables measured both before and after the intervention period. Differences in response between the vastus lateralis, rectus femoris, and vastus intermedius were analyzed with one-way ANOVA and Tukey's multiple comparisons test. Associations between variables were investigated using Pearson's correlation coefficient. Correlations were considered weak if $r<0.4$, moderate if $r=0.4-0.6$, and strong if $r>0.6$. Statistical analyses were performed using GraphPad Prism6 software (GraphPad Software, Inc., La Jolla, CA). Twosided statistical significance was set at $P<0.05$.

\section{Results}

There were no significant differences between ST and $\mathrm{CON}$ in physical characteristics or protein intake at baseline, although ST tended to be older than CON (Table 2). All 22 subjects completed the study. However, one subject in CON was excluded from the per-protocol analysis, as the subject started strength training during the intervention period. Thus, 11 subjects in ST and 10 subjects in CON remained for the per-protocol analyses. Moreover, one additional subject in CON did not perform the ultrasound measurement after the intervention. Thus, for this analysis, the number of subjects in ST and CON is 11 and 9, respectively. No adverse events occurred in any of the groups.

\section{Baseline correlations}

A strong correlation was observed between leg lean mass and knee extension $1 \mathrm{RM}$ at baseline $(r=0.80, P<0.001)$. In addition, 1RM strength normalized to body mass showed moderate correlations with habitual gait velocity $(r=0.46, P<0.05)$ and stair-climbing time $(r=-0.50$, $P<0.05)$, but not with five times chair rise $(r=-0.25, P$ $=0.26)($ Table 3$)$.

Table 2 Baseline participant characteristics

\begin{tabular}{|c|c|c|c|}
\hline & $\begin{array}{l}\mathrm{ST}(n=11) \\
n \text { or Mean } \pm \mathrm{SD}\end{array}$ & $\begin{array}{l}\mathrm{CON}(n=11) \\
n \text { or Mean } \pm \mathrm{SD}\end{array}$ & $P$ value \\
\hline Men/women $(n)$ & $4 / 7$ & $3 / 8$ & \\
\hline Age & $86.6 \pm 6.0$ & $82.6 \pm 4.5$ & 0.09 \\
\hline Height (m) & $1.68 \pm 0.07$ & $1.65 \pm 0.10$ & 0.50 \\
\hline Body mass (kg) & $73.6 \pm 12.7$ & $71.5 \pm 9.5$ & 0.67 \\
\hline Lean mass $(\mathrm{kg})$ & $44.8 \pm 5.6$ & $41.5 \pm 4.5$ & 0.14 \\
\hline $\begin{array}{l}\text { BMI (body mass/ } \\
\text { height }^{2} \text { ) }\end{array}$ & $26.1 \pm 4.2$ & $26.3 \pm 3.8$ & 0.94 \\
\hline $\begin{array}{l}\text { Protein intake }(\mathrm{g} / \mathrm{kg} / \\
\text { day) }\end{array}$ & $0.91 \pm 0.17$ & $0.83 \pm 0.29$ & 0.47 \\
\hline $\begin{array}{l}\text { Short physical perfor- } \\
\text { mance battery (SPPB) }\end{array}$ & $9.0 \pm 1.4$ & $8.9 \pm 2.2$ & 0.91 \\
\hline
\end{tabular}


Table 3 Correlations at baseline for all participants $(n=22)$

\begin{tabular}{llllllll}
\hline & Measure & 1 & 2 & 3 & 4 & 5 & 6 \\
\hline 1. & Leg lean mass (kg) & - & & & & \\
2. & Vastus lateralis thickness (mm) & 0.29 & - & & & \\
3. & Knee extension 1 RM (kg) & $0.80^{* * *}$ & 0.22 & - & & \\
4. & Habitual gait velocity (m/s) & 0.25 & 0.13 & $0.46^{*}$ & - & & \\
5. & Five times chair rise (s) & -0.02 & 0.02 & -0.25 & $-0.54 * *$ & - & \\
6. & Stair climbing (s) & -0.40 & 0.01 & $-0.50^{*}$ & $-0.69 * * *$ & $0.53^{*}$ & - \\
\hline
\end{tabular}

Values for leg lean mass, vastus lateralis thickness, and knee extension 1 RM were normalized to body mass prior to correlation analyses

$* P<0.05, * * P<0.01, * * * P<0.001$

\section{Adherence}

All subjects in ST performed at least $90 \%$ of the training sessions in the 10 -week period $(95.8 \pm 2.6)$, and all subjects in ST were therefore included in the per-protocol analysis. There were no correlations between adherence and training effect on any variable.

\section{Protein intake}

ST and CON had a protein intake of $0.91( \pm 0.17)$ and 0.83 $( \pm 0.29) \mathrm{g} \mathrm{kg}^{-1} \mathrm{day}^{-1}$ at baseline, respectively. As a consequence of the protein supplement, ST increased their daily protein intake to $1.22 \mathrm{~g} \mathrm{~kg}^{-1} \mathrm{day}^{-1}(P=0.002)$. No significant change was observed in $\mathrm{CON}$, and the group $\times$ time interaction only tended to be significant $(P=0.058)$ (Fig. 2).

\section{Body composition}

Body mass tended to increase from baseline in $\mathrm{CON}$ $(1.1 \pm 1.5 \mathrm{~kg}, P=0.056)$, but not in $\mathrm{ST}(0.7 \pm 1.5 \mathrm{~kg}$, $P=0.24)$. No group $\times$ time interaction was observed $(P=0.54)$. However, a group $\times$ time interaction was observed for leg lean mass $(P=0.001)$, due to an increase from baseline in $\mathrm{ST}(0.7 \pm 0.3 \mathrm{~kg}, P<0.001)$, and no change in CON $(0.1 \pm 0.3 \mathrm{~kg}, P=0.69)$ (Fig. 3a). For fat mass, a tendency toward an increase was observed in CON $(0.6 \pm 0.7 \mathrm{~kg}$; $P=0.052$ ), whereas a non-significant reduction was observed in ST $(-0.5 \pm 0.9 \mathrm{~kg}, P=0.15)$. Thus, for fat mass, a group $\times$ time interaction was observed $(P=0.007)$ (Fig. 3b).

\section{Muscle thickness}

In ST, increased thickness of vastus lateralis $(4.4 \pm 3.2 \%)$, rectus femoris $(6.7 \pm 5.1 \%)$, and vastus intermedius $(5.8 \pm 5.9 \%)$ was observed $(P<0.001$ for all muscles $)$ (Fig. 4). Muscle thickness did not change in $\mathrm{CON}$, and a group $\times$ time interaction was observed for all muscles investigated $(P<0.01$ for all muscles $)$. There were no differences between the relative change in vastus lateralis, rectus femoris, and vastus intermedius in $\mathrm{ST}(P=0.38)$. A strong correlation was observed between change in vastus lateralis thickness and the change in leg lean mass in the training group $(r=0.67, P<0.05)$.
(A)

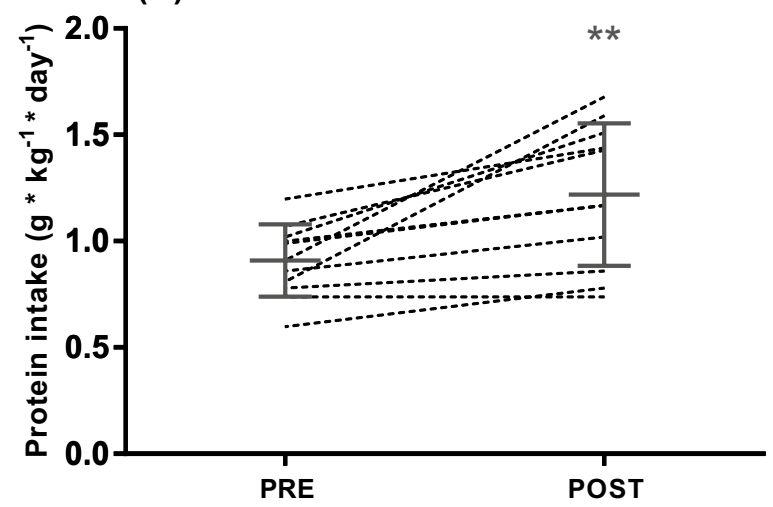

(B)

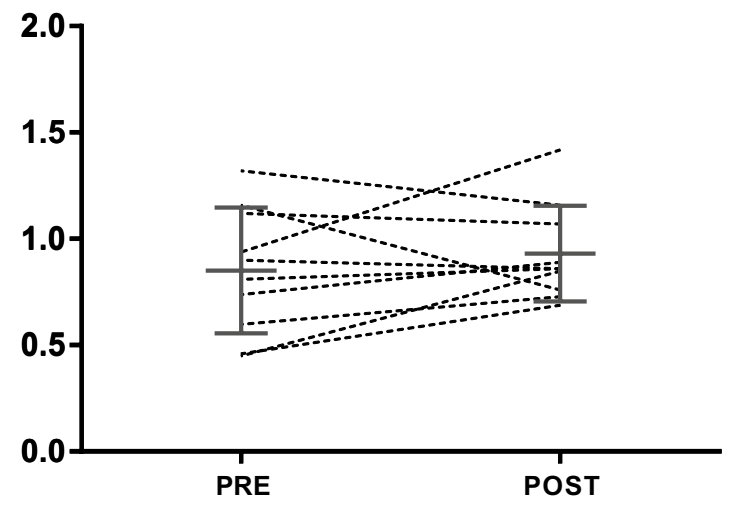

Fig. 2 Change in daily protein intake in $\mathrm{ST}(\mathbf{a})$ and $\mathrm{CON}(\mathbf{b}) . * * P<0.01$, compared to baseline within the group 
(A)

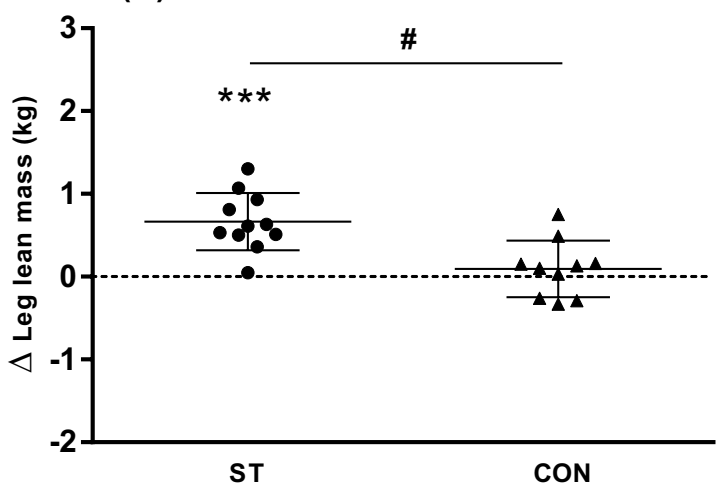

(B)

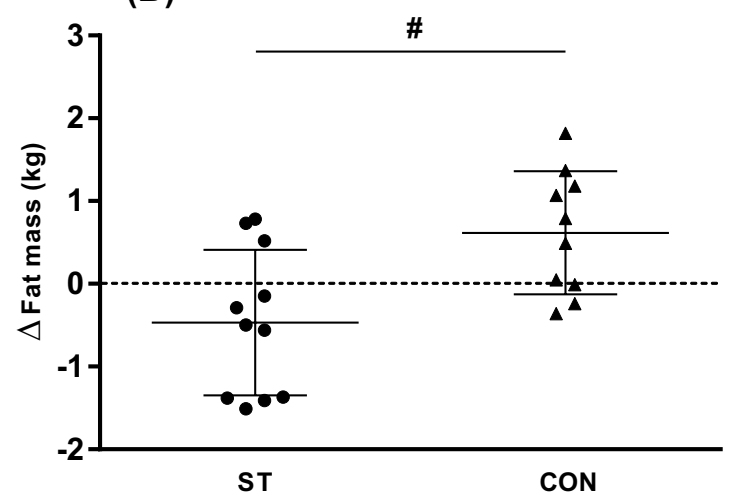

Fig. 3 Change in leg lean mass (a) and total body fat mass (b) in ST $(\bullet), \operatorname{CON}(\mathbf{\Delta}) .{ }^{\#} P<0.05$, group $\times$ time interaction. $* * * P<0.001$, compared to baseline within group. Results are presented as individual values and with mean and standard deviation (SD) for each group

\section{Muscle strength}

ST improved KE $1 \mathrm{RM}$ and isometric MVC by $23 \pm 15 \%$ and $20 \pm 11 \%$, respectively, and a group $\times$ time interaction was observed for both tests $(P<0.001)$ (Fig. 5). The change in knee extension 1RM was not correlated with the change in leg lean mass, or with the change in thickness of vastus lateralis, rectus femoris, or vastus intermedius. Force at $100 \mathrm{~ms}$ during the MVC improved from baseline only in ST $(22 \pm 22 \%)$, and a group $\times$ time interaction was observed $(P=0.039)$. Relative rate of torque development $\left(\mathrm{RTD}_{\max } I\right.$ maximal torque) did not change in either group.

\section{Functional performance}

ST participants used less time on five times chair rise and stair climbing after the intervention, with improvements of $10 \pm 13(P=0.05)$ and $11 \pm 12 \%(P=0.034)$ from baseline, respectively (Fig. 6). A group $\times$ time interaction was observed for stair climbing $(P=0.019)$, but not five times chair rise $(P=0.13)$. In addition, a group $\times$ time interaction was observed for habitual gait velocity $(P=0.028)$, due to a non-significant improvement in ST $(7 \pm 10 \% ; P=0.17)$, along with a non-significant decline in CON $(-5 \pm 14 \%$, $P=0.24$ ) (Fig. 7). No significant correlations were observed between training-induced changes in knee extension 1RM and changes in five times chair-rise time $(r=-0.46$, $P=0.15)$ or stair-climbing time $(r=0.04, P=0.89)$. However, the change in knee extension 1RM tended to correlate with the change in habitual gait velocity $(r=0.54, P=0.09)$. Furthermore, a strong correlation was observed between the change in isometric MVC and the change in habitual gait velocity in the training group ( $r=0.70, P=0.015)$ (Fig. 8). No significant correlations were observed between the improvement in force at $100 \mathrm{~ms}$ during MVC and changes in functional performance.

\section{Discussion}

Thirty minutes of heavy-load strength training performed three times per week in combination with protein supplementation increased leg lean mass, as well as strength and functional capacity in mobility-limited older adults. Concerning hypertrophy of the vastus lateralis, rectus femoris and vastus intermedius, we found no significant differences between the muscles. Although no correlations were observed between the changes in muscle mass/thickness and changes in muscle strength, we did observe a correlations between strength gains and improvements in functional capacity.

\section{Leg lean mass and muscle thickness}

The intervention was successful in eliciting muscle hypertrophy, demonstrated both by the change in leg lean mass and muscle thickness. A meta-analysis of 49 studies, representing a total of 1328 participants, examined the effect of whole-body strength training on lean body mass (LBM) in individuals $>50$ years. The mean intervention duration was 20.5 weeks, and the weighted pooled estimate of lean body mass change was $1.1 \mathrm{~kg}$ [12]. Interestingly, the metaanalysis also found a negative association between age and change in lean mass. With a mean age of 86.6 years, the subjects in our experimental group (ST) can be categorized as very old, and thus an increase of $0.7 \mathrm{~kg}$ in leg lean mass after only 10 weeks of leg exercise is somewhat higher than expected. Due to the design of our study, we were not able to conclude whether the protein supplement contributed to the observed effects. The literature is equivocal with regard to the importance of protein supplementation during strength training in healthy old individuals $[3,6,13,14]$, but there are reports of an additive effect in the frail elderly [10]. According to the diet assessments, the experimental group had a 


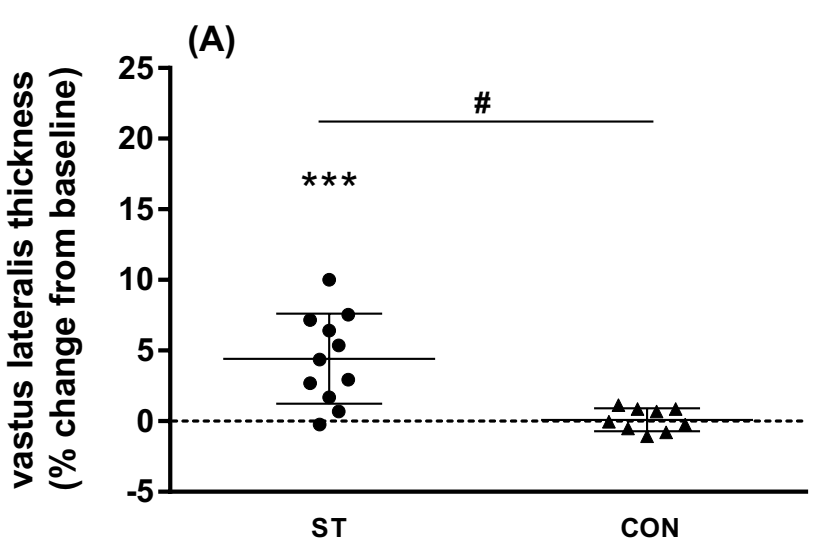

(B)
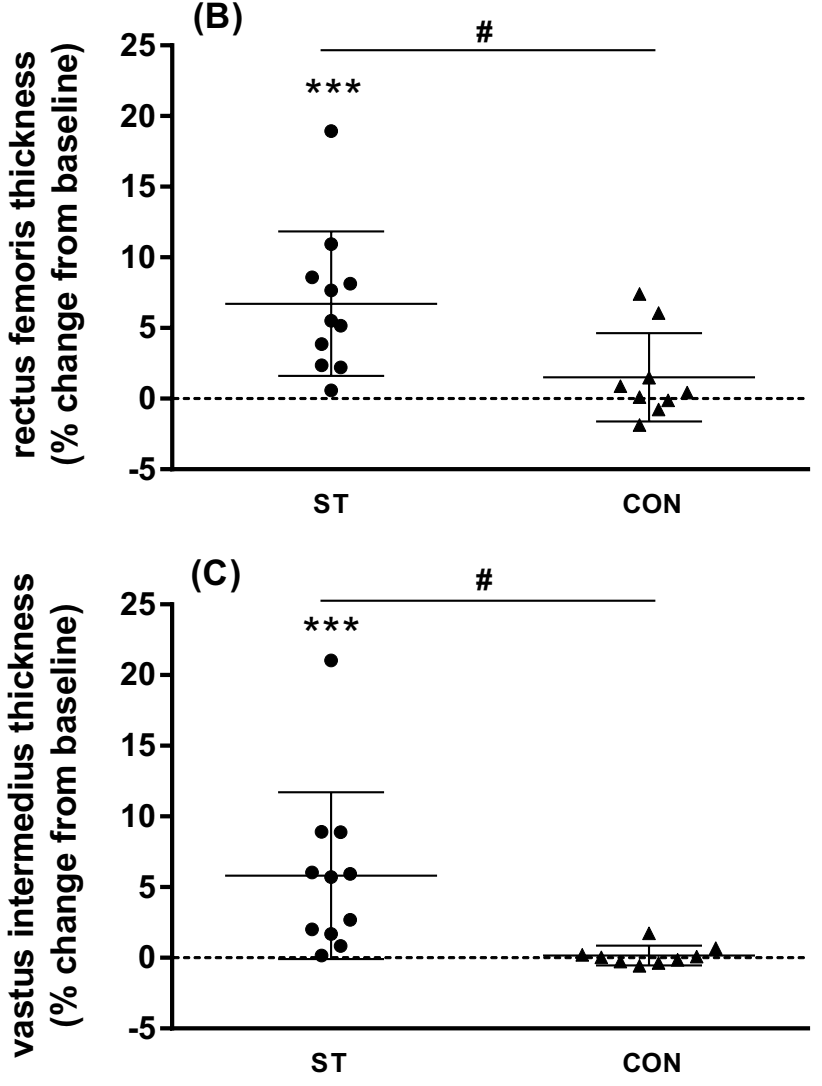

Fig. 4 Percentage changes in ST $(\bullet)$ and CON $(\boldsymbol{\Delta})$, measured in the vastus lateralis (a), rectus femoris (b), and vastus intermedius (c). ${ }^{\#} P<0.01$, group $\times$ time interaction. $* * * P<0.001$, compared to baseline within group. Results are presented as individual values and with mean and standard deviation (SD) for each group

protein intake of $0.91 \mathrm{~g} \mathrm{~kg}^{-1} \mathrm{day}^{-1}$ at baseline. Although this is above the recommended dietary allowance (RDA) of $0.8 \mathrm{~g} \mathrm{~kg}^{-1} \mathrm{day}^{-1}$ [15], it is below the Nordic recommendations of $1.2 \mathrm{~g} \mathrm{~kg}^{-1} \mathrm{day}^{-1}$ for older adults [16]. Furthermore, the variability in protein intake at baseline was quite high (range: $0.6-1.2 \mathrm{~g} \mathrm{~kg}^{-1} \mathrm{day}^{-1}$ ), and it is therefore possible that the protein supplement had additive effects at least in the individuals with a low protein intake at baseline.

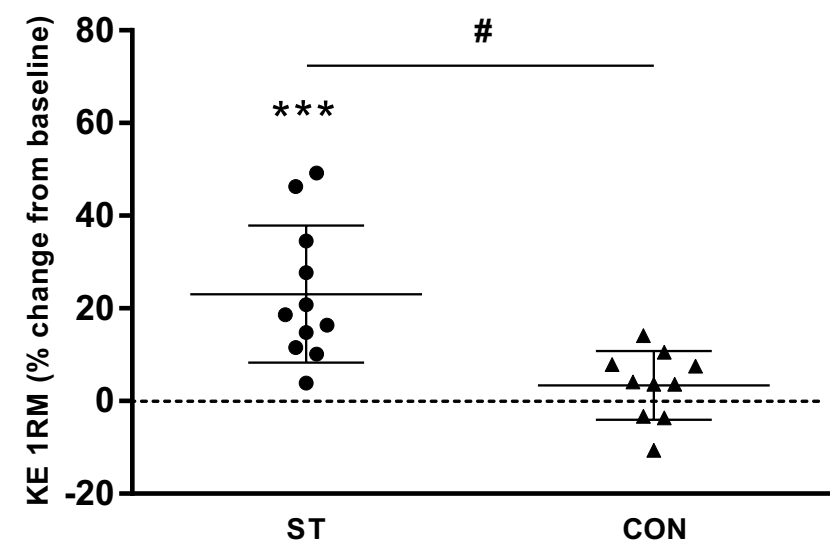

Fig. 5 Percentage change in knee extension $1 \mathrm{RM}$ in ST $(\bullet)$ and CON ( $\boldsymbol{\Delta}) .{ }^{\#} P<0.001$, group $\times$ time interaction. $* * * P<0.001$, compared to baseline within group. Results are presented as individual values and with mean and standard deviation (SD) for each group

Muscle thickness of the vastus lateralis, rectus femoris, and vastus intermedius increased by $4.4,6.7$, and $5.8 \%$, respectively. In a systematic review, Stewart and coworkers investigated the hypertrophic response to strength training in very old individuals ( $>75$ years) [17]. Three of four studies observed increased cross-sectional area (CSA) of the quadriceps femoris, reporting improvements of 2-9\%. Moreover, a recent study observed a $3.4 \%$ increase in quadriceps femoris CSA following 12 weeks of heavy-load strength training and protein supplementation in very old subjects (83-94 years) [7]. As we measured muscle thickness and not cross-sectional area, we cannot directly compare our results with the above findings. However, a 7.5\% increase in the vastus lateralis thickness assessed by ultrasound corresponded to a $5.2 \%$ increase in CSA assessed by MRI following strength training in young individuals [18]. Accordingly, our observation of 4-7\% increases in muscle thickness corresponds well with previous reports $[6,7,9,19]$. No significant differences between the vastus lateralis, rectus femoris, and vastus intermedius were observed in the present investigation. To our knowledge, no study has investigated the relative hypertrophy in different compartments of quadriceps femoris in this population previously. In somewhat younger individuals ( $67 \pm 5$ years), increases of $6 \%$ and $16 \%$ were observed for the vastus lateralis and rectus femoris, respectively, after 12 weeks of strength training [20]. Also in young individuals, greater increases have been observed for the rectus femoris $(\sim 18 \%)$, compared to both vastus lateralis $(\sim 8 \%)$ and vastus intermedius ( 9\%) [21]. Although direct comparisons across studies should be performed with care, the overall greater improvements in muscle thickness in young [21], as well as in individuals in their late 60s [20], imply that the hypertrophic response to strength training may indeed be reduced at very high age, in line with other reports $[12,22$, 
(A)

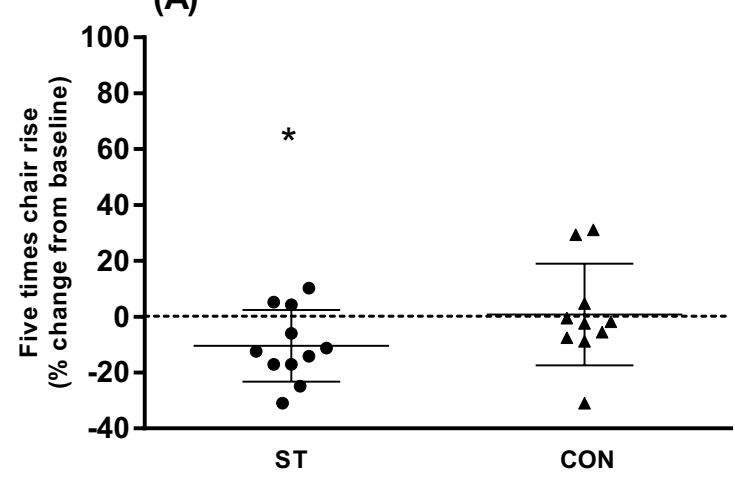

Fig. 6 Percentage change in time used during five times chair rise (a) and stair climbing (b) in $\mathrm{ST}(\bullet)$ and $\operatorname{CON}(\boldsymbol{\Delta}) .{ }^{\#} P<0.05$, group $\times$ time interaction. $* P<0.05$, compared to baseline within the group. Results are presented as individual values and with mean and

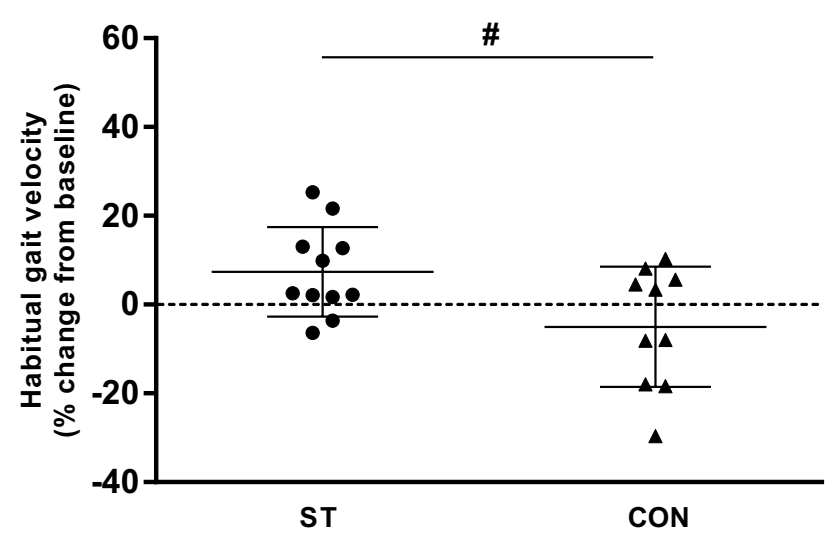

Fig. 7 Percentage change in habitual gait velocity in ST $(\bullet)$ and CON $(\boldsymbol{\Lambda}) .{ }^{\#} P<0.05$, group $\times$ time interaction. Results are presented as individual values and with mean and standard deviation (SD) for each group. Data were log transformed prior to statistical analyses

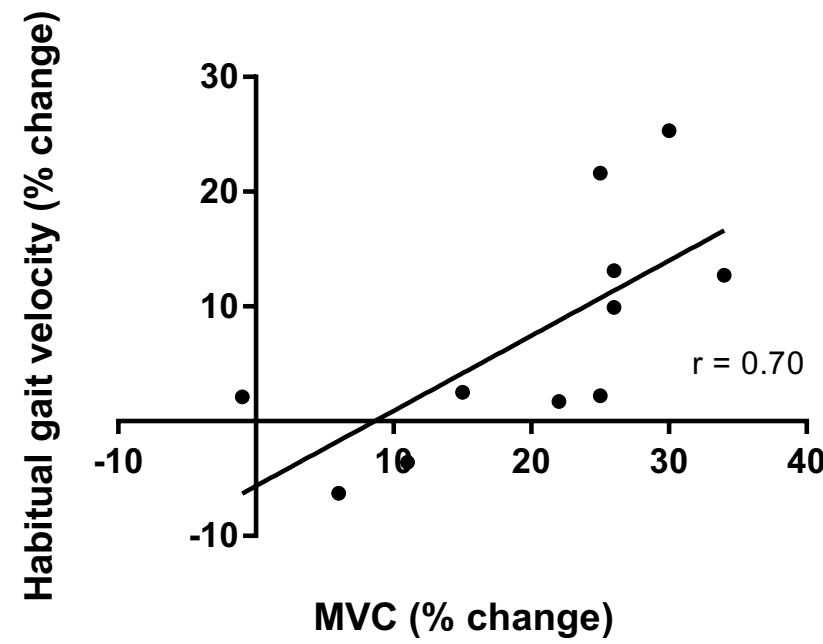

Fig. 8 Relationship between the change in knee extension MVC and habitual gait velocity in ST
(B)

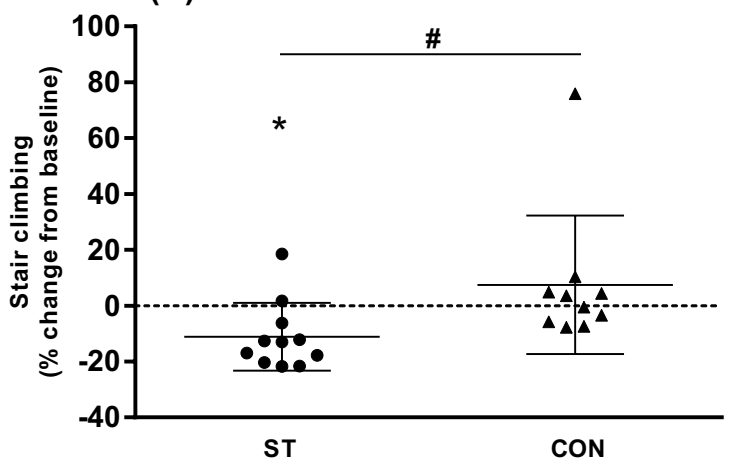

standard deviation (SD) for each group. Data were log transformed prior to statistical analyses. The group $\times$ time interaction in B was significant $(P=0.017)$ even if the outlier in $\mathrm{CON}$ was excluded from the analysis

23]. Additionally, our results suggest that the vastus lateralis, rectus femoris, and vastus intermedius in relative terms contribute equally to the strength training-induced increase in quadriceps femoris area in this population.

\section{Muscle strength and rate of torque development}

Knee extension $1 \mathrm{RM}$ increased by $23 \%$ in the training group. This is in the lower range of previous reports in similar populations $(\sim 25$ to $\sim 200 \%)[6,7,9,24]$. In the present investigation, testing and training were performed in separate machines. This is likely advantageous to limit the learning effects, which may have contributed to the substantial 1RM strength gains in some of the previous reports. This is supported by the often-observed discrepancy between increases in dynamic (1RM) and isometric (MVC) strength [7, 8]. In contrast, dynamic and isometric strength gains were in good agreement in the present study, suggesting that the $23 \%$ improvement in knee extension 1RM was mainly due to an increase in the force-generating capacity of the quadriceps femoris muscles, and not due to learning effects. However, in line with our hypothesis, strength gains still exceeded the increases in leg lean mass (4\%) and muscle thickness $(4-7 \%)$. The latter imply that additional factors contributed to the improvements in strength. In this regard, increased activation level, muscle density, muscle length, and single fiber-specific force may all have contributed $[19,25,26]$. Although not significant, we did observe associations between the change in knee extension 1RM and habitual gait velocity $(r=0.54, P=0.09)$ and time used during five times chair rise $(r=-0.46, P=0.15)$. Additionally, the change in isometric MVC was significantly correlated with the change in habitual gait velocity 
$(r=0.70, P<0.05)$. These relationships suggest that the extent to which strength gains are achieved is decisive with respect to the degree of functional improvement in this population.

The improvements of knee extension 1RM and peak MVC were paralleled by improvement of torque at $100 \mathrm{~ms}$ during MVC. Torque at $100 \mathrm{~ms}$ represents the capacity of the muscle as a whole to produce force rapidly, and is therefore an absolute measure of rate of torque development (RTD). However, if torque at $100 \mathrm{~ms}$ were normalized to maximal torque, no effect of training was observed. Moreover, normalized $\mathrm{RTD}_{\max }$, given as the rise in torque development relative to maximal torque, did not improve. Hence, both these relative measures of RTD suggest that the intrinsic properties of the knee extensors were not affected. In theory, it would not be surprising if we had observed increased relative RTD as well, due to the preferential hypertrophy of type II fibers with strength training [27-29]. Nevertheless, increasing muscle strength alone will allow the muscle to produce more force over a short time interval, and thus improve the muscle's ability to produce power. This is important, since functional performance correlates even better with muscle power than muscle strength [30,31]. In addition, improved ability to produce force rapidly may prevent falls, due to an increased ability to correct unexpected imbalances that might easily occur during daily life (e.g., walking) [32].

\section{Functional performance}

The control subjects in the present investigation displayed a non-significant reduction $(5 \%)$ in gait velocity over the 10 -week period, indicating that functional declines in this population are rapid. It has been shown previously that gait velocity is associated with survival in older adults $[33,34]$, underscoring the importance of countermeasures. The 7\% improvement observed in the training group is in agreement with two meta-analyses showing improvements of approximately $5-10 \%$ following strength training in older adults $[35,36]$. The training group also improved five times chair-rise and stair-climbing performance by 10 and $11 \%$, respectively, again in accordance with previous reports [5, 6, 24, 37, 38]. It has been suggested that highspeed power training has some advantages over traditional strength training with regard to improvements in function in this population [39]. Nevertheless, our findings demonstrate that traditional heavy-load strength training effectively improves functional performance in mobility-limited older adults. Importantly, no adverse event occurred and adherence to the training was high, demonstrating that such training can be effectively and safely implemented.

\section{Limitations}

With only two groups, where the intervention group both performed strength training and received protein supplementation, we were not able to discern the effects from training and from supplementation. Including a protein only and training only group would have provided this information. Moreover, intention-to-treat analyses were not performed in the present investigation. Although we aimed to recruit 26 subjects to account for dropouts, we were only able to recruit 22 participants. Luckily, as there were no dropouts, we still reached the number needed based on the power calculation. Another limitation of the study is that subjects and test personnel were not blinded (except ultrasound analysis). The lack of information about compliance to the supplement also represents a limitation of the study.

\section{Conclusions}

In line with our hypothesis, strength gains (20-23\%) exceeded increases in leg lean mass (4\%) and muscle thickness $(4-7 \%)$ in mobility-limited older adults following heavy-load strength training. We nevertheless hypothesized that the training-induced hypertrophy would correlate with increases in isometric strength, but no such correlations were observed. Collectively, these findings indicate that other factors (e.g., activation level and muscle quality) contributed to the increases in strength. In line with our hypothesis, increases in muscle strength correlated with functional improvements, suggesting that interventions eliciting large improvements in strength may also be superior in terms of functional gains in this group vulnerable to frailty and functional decline.

\section{Perspectives}

Among older adults with mobility limitations living at home, many are at the verge of being institutionalized. Increasing functional capacity through strength training may allow these individuals to become less dependent on assistance in various daily activities, to an extent where their activity level can be better maintained, and further loss of muscle mass and strength can be blunted or even prevented. Such strategies are important both from a socioeconomic and individual perspective. Future studies should address the mechanisms underlying the often-observed mismatch between improvements in muscle strength and size. A deeper understanding of such factors will strengthen the basis for the development of optimal countermeasures. 
Acknowledgements The authors would like to thank Kristin Holte for analysis of the dietary registrations, Mauritz Kaashagen for performing the ultrasound measurements, and Anne Lene Nordengen for assistance during the supervision of training. We thank TINE SA for providing the nutritional supplements. We also thank our participants for their great contributions.

Author contributions SA and TR contributed to the conception and design of the research; SA performed the experiments; SA analyzed the data; SA, OS, HB, and TR interpreted the results of the experiments; SA prepared the figures; SA drafted the manuscript; SA, OS, HB, and TR edited and revised the manuscript; SA, OS, HB, and TR approved the final version of the manuscript.

Funding The study was funded by the Norwegian School of Sport Sciences.

\section{Compliance with ethical standards}

Conflict of interest The authors declare that there is no conflict of interest regarding the publication of this article.

Ethical approval All procedures were in accordance with the ethical standards of the national research committee (Regional Committee for Medical and Health Research Ethics, Section C, South East Norway) and the 1964 Helsinki declaration and its later amendments.

Informed consent Informed consent was obtained from all individual participants included in the study.

\section{References}

1. Hayashida I, Tanimoto Y, Takahashi Y et al (2014) Correlation between muscle strength and muscle mass, and their association with walking speed, in community-dwelling elderly Japanese individuals. PLoS One 9:e111810

2. Vasunilashorn S, Coppin AK, Patel KV et al (2009) Use of the short physical performance battery score to predict loss of ability to walk 400 meters: analysis from the InCHIANTI study. J Gerontol Ser A Biol Sci Med Sci 64:223-229

3. Cermak NM, Res PT, de Groot LC et al (2012) Protein supplementation augments the adaptive response of skeletal muscle to resistance-type exercise training: a meta-analysis. Am J Clin Nutr 96:1454-1464

4. Verdijk LB, Jonkers RA, Gleeson BG et al (2009) Protein supplementation before and after exercise does not further augment skeletal muscle hypertrophy after resistance training in elderly men. Am J Clin Nutr 89:608-616

5. Pinto RS, Correa CS, Radaelli R et al (2014) Short-term strength training improves muscle quality and functional capacity of elderly women. Age 36(1):365-372

6. Chale A, Cloutier GJ, Hau C et al (2013) Efficacy of whey protein supplementation on resistance exercise-induced changes in lean mass, muscle strength, and physical function in mobility-limited older adults. J Gerontol Ser A Biol Sci Med Sci 68:682-690

7. Bechshoft RL, Malmgaard-Clausen NM, Gliese B et al (2017) Improved skeletal muscle mass and strength after heavy strength training in very old individuals. Exp Gerontol 92:96-105

8. Harridge SD, Kryger A, Stensgaard A (1999) Knee extensor strength, activation, and size in very elderly people following strength training. Muscle Nerve 22:831-839
9. Fiatarone MA, O'Neill EF, Ryan ND et al (1994) Exercise training and nutritional supplementation for physical frailty in very elderly people. N Engl J Med 330:1769-1775

10. Tieland M, Dirks ML, van der Zwaluw N et al (2012) Protein supplementation increases muscle mass gain during prolonged resistance-type exercise training in frail elderly people: a randomized, double-blind, placebo-controlled trial. J Am Med Dir Assoc 13:713-719

11. Guralnik JM, Ferrucci L, Simonsick EM et al (1995) Lowerextremity function in persons over the age of 70 years as a predictor of subsequent disability. N Engl J Med 332:556-561

12. Peterson MD, Sen A, Gordon PM (2011) Influence of resistance exercise on lean body mass in aging adults: a meta-analysis. Med Sci Sports Exerc 43:249-258

13. Thomas DK, Quinn MA, Saunders DH et al (2016) Protein supplementation does not significantly augment the effects of resistance exercise training in older adults: a systematic review. J Am Med Dir Assoc 17:959.e1-959.e9

14. Finger D, Goltz FR, Umpierre D et al (2015) Effects of protein supplementation in older adults undergoing resistance training: a systematic review and meta-analysis. Sports Med 45:245-255

15. Volpi E, Campbell WW, Dwyer JT et al (2013) Is the optimal level of protein intake for older adults greater than the recommended dietary allowance? J Gerontol Ser A Biol Sci Med Sci 68:677-681

16. Nordic Nutrition Recommendations (2012) Integrating nutrition and physical activity, 5th edn. Nordic Counsil of Ministers; 2014, Copenhagen, Denmark

17. Stewart VH, Saunders DH, Greig CA (2014) Responsiveness of muscle size and strength to physical training in very elderly people: a systematic review. Scand J Med Sci Sports 24:e1-e10

18. Franchi MV, Longo S, Mallinson J et al (2018) Muscle thickness correlates to muscle cross-sectional area in the assessment of strength training-induced hypertrophy. Scand J Med Sci Sports 28:846-853

19. Sipila S, Suominen H (1995) Effects of strength and endurance training on thigh and leg muscle mass and composition in elderly women. J Appl Physiol 78:334-340

20. Bjornsen T, Salvesen S, Berntsen S et al (2016) Vitamin C and E supplementation blunts increases in total lean body mass in elderly men after strength training. Scand J Med Sci Sports 26:755-763

21. Ema R, Wakahara T, Miyamoto N et al (2013) Inhomogeneous architectural changes of the quadriceps femoris induced by resistance training. Eur J Appl Physiol 113:2691-2703

22. Greig CA, Gray C, Rankin D et al (2011) Blunting of adaptive responses to resistance exercise training in women over $75 y$. Exp Gerontol 46:884-890

23. Raue U, Slivka D, Minchev K et al (2009) Improvements in whole muscle and myocellular function are limited with high-intensity resistance training in octogenarian women. J Appl Physiol 106:1611-1617

24. Seynnes O, Fiatarone Singh MA, Hue O et al (2004) Physiological and functional responses to low-moderate versus high-intensity progressive resistance training in frail elders. J Gerontol Ser A Biol Sci Med Sci 59:503-509

25. Arnold P, Bautmans I (2014) The influence of strength training on muscle activation in elderly persons: a systematic review and meta-analysis. Exp Gerontol 58:58-68

26. Frontera WR, Hughes VA, Krivickas LS et al (2003) Strength training in older women: early and late changes in whole muscle and single cells. Muscle Nerve 28:601-608

27. Verdijk LB, Gleeson BG, Jonkers RA et al (2009) Skeletal muscle hypertrophy following resistance training is accompanied by a fiber type-specific increase in satellite cell content in elderly men. J Gerontol Ser A Biol Sci Med Sci 64:332-339 
28. Kosek DJ, Kim JS, Petrella JK et al (2006) Efficacy of 3 days/week resistance training on myofiber hypertrophy and myogenic mechanisms in young vs. older adults. J Appl Physiol 101:531-544

29. Kryger AI, Andersen JL (2007) Resistance training in the oldest old: consequences for muscle strength, fiber types, fiber size, and MHC isoforms. Scand J Med Sci Sports 17:422-430

30. Reid KF, Fielding RA (2012) Skeletal muscle power: a critical determinant of physical functioning in older adults. Exerc Sport Sci Rev 40:4-12

31. Bean JF, Kiely DK, Herman S et al (2002) The relationship between leg power and physical performance in mobility-limited older people. J Am Geriatr Soc 50:461-467

32. Pijnappels M, Reeves ND, Maganaris CN et al (2008) Tripping without falling; lower limb strength, a limitation for balance recovery and a target for training in the elderly. J Electromyogr Kinesiol 18:188-196

33. White DK, Neogi T, Nevitt MC et al (2013) Trajectories of gait speed predict mortality in well-functioning older adults: the health, aging and body composition study. J Gerontol Ser A Biol Sci Med Sci 68:456-464

34. Studenski S, Perera S, Patel K et al (2011) Gait speed and survival in older adults. JAMA 305:50-58
35. Van Abbema R, De Greef M, Craje C et al (2015) What type, or combination of exercise can improve preferred gait speed in older adults? A Meta Anal BMC Geriatr 15:72

36. Hortobagyi T, Lesinski M, Gabler M et al (2015) Effects of three types of exercise interventions on healthy old adults' gait speed: a systematic review and meta-analysis. Sports Med 45:1627-1643

37. Schot PK, Knutzen KM, Poole SM et al (2003) Sit-to-stand performance of older adults following strength training. Res Q Exerc Sport $74: 1-8$

38. Dias CP, Toscan R, de Camargo M et al (2015) Effects of eccentric-focused and conventional resistance training on strength and functional capacity of older adults. Age 37:99

39. Sayers SP, Gibson K (2014) High-speed power training in older adults: a shift of the external resistance at which peak power is produced. J Strength Cond Res Natl Strength Cond Assoc 28:616-621

Publisher's Note Springer Nature remains neutral with regard to jurisdictional claims in published maps and institutional affiliations. 